\title{
Dopamine-functionalized InP/ZnS quantum dots as fluorescence probes for the detection of adenosine in microfluidic chip
}

This article was published in the following Dove Press journal:

International Journal of Nanomedicine

25 August 2015

Number of times this article has been viewed

\section{Seshadri Reddy Ankireddy Jongsung Kim}

Department of Chemical and Biological Engineering, Gachon University, Seongnam, Gyeonggi-Do, South Korea
Correspondence: Jongsung Kim

Department of Chemical and Biological

Engineering, Gachon University,

Seongnam, Gyeonggi-Do 46I-70I,

South Korea

Email jongkim@gachon.ac.kr
Abstract: Microbeads are frequently used as solid supports for biomolecules such as proteins and nucleic acids in heterogeneous microfluidic assays. Chip-based, quantum dot (QD)-beadbiomolecule probes have been used for the detection of various types of DNA. In this study, we developed dopamine (DA)-functionalized InP/ZnS QDs (QDs-DA) as fluorescence probes for the detection of adenosine in microfluidic chips. The photoluminescence (PL) intensity of the QDs-DA is quenched by $\mathrm{Zn}^{2+}$ because of the strong coordination interactions. In the presence of adenosine, $\mathrm{Zn}^{2+}$ cations preferentially bind to adenosine, and the PL intensity of the QDs-DA is recovered. A polydimethylsiloxane-based microfluidic chip was fabricated, and adenosine detection was confirmed using QDs-DA probes.

Keywords: quantum dots, photoluminescence, microfluidic chip, adenosine, mercaptopropionic acid, mercaptoundecanoic acid, PDMS

\section{Introduction}

Adenosine is an inhibitory neurotransmitter and a signaling molecule that is involved in energy production in the form of adenosine triphosphate (ATP) at the molecular level. Adenosine signaling was first discovered in the beginning of the 20th century, and it is known to regulate innumerable physiological functions within animal cells. Adenosine also plays a key role in chemical neurotransmission ${ }^{1}$ and in the control of coronary blood flow in human beings. The effects of adenosine at the cellular level are complicated, because its mediation can be either excitatory or inhibitory. Adenosine is also used to treat supraventricular tachycardia, which is cardiac arrhythmia caused by faulty electrical functioning of the heart. ${ }^{2}$

Over the past two decades, several analytical methods have been developed for quantifying adenosine, including potentiometry, colorimetry, ${ }^{3}$ electrochemical methods, ${ }^{4}$ high-performance liquid chromatography with fluorescence detection, ${ }^{5,6}$ and capillary electrophoresis. ${ }^{7}$ All these methods have several drawbacks, including poor sensitivity, poor detection limits, difficulties with sample separation, and expensive instrumentation. Photoluminescence (PL) spectroscopy has high sensitivity and a wide linear working range, and the instrumentation is simple and inexpensive; it has therefore become an attractive analytical tool for the analysis of pharmaceutical and biological samples.

Polydimethylsiloxane (PDMS) is a biocompatible amorphous polymer ${ }^{8}$ and is commonly used as a drug-delivery vehicle in medicine. Recently, reactive plasmas have been developed to enhance the adhesion of PDMS on glass slides and the immobilization of biomolecules. The chemical and topographical properties of the exposed surfaces can be modified. Several studies have been performed on surface modifications of 
PDMS using plasma treatment. ${ }^{9}$ Microfluidic techniques are attracting increased attention because of their advantages over macroscopic instrumentation, eg, small amounts of samples and reagents are needed, and the techniques are faster and more accurate. Recently, the integration of efficient sample handling by microfluidics and microbead-based arrays has been reported for multitarget detection, providing an ideal platform for sensitive detection of disease-related biomolecules and neurotransmitters. ${ }^{10} \mathrm{~A}$ microfluidic bead-based platform has been developed that requires smaller amounts of samples and gives reaction times of a few minutes rather than several hours. ${ }^{11}$

Dopamine (DA), which is neurotransmitter belonging to the catecholamine family, plays an important role in the human brain and body. DA is released by nerve cells as a chemical messenger to send signals between neurons. The brain includes several distinct DA systems, one of which plays a major role in reward-motivated behavior, and these DA systems mediate the enhancement of DA neuronal activity in nerve cells. ${ }^{12,13} \mathrm{CdSe}$, $\mathrm{CdS}$, and CdTe quantum dots (QDs) have several advantages, such as high photoluminescence quantum efficiency and sizetunable absorption and photoluminescence in the visible to Near-infrared (NIR) region, yet concerns about their toxicity due to the presence of cadmium are to be resolved. ${ }^{14}$ Compared with Cd-based QDs, InP QDs are nontoxic and promising for in vivo imaging and clinical application in medicine field and photodynamic cancer therapy. Although QDs based on $\mathrm{Pb}$ and $\mathrm{Hg}$ core or shell show NIR absorption and photoluminescence characteristics, those are less promising for biological applications due to the presence of toxic heavy metals. Thus, we excluded $\mathrm{Pb}$-, Cd-, and $\mathrm{Hg}$-based QDs and focused on the synthesis of $\mathrm{InP} / \mathrm{ZnS}$ core and shell QDs that are being widely applied in clinical applications. ${ }^{15-17}$

Recently, $\mathrm{Ni}^{2+}$-imidazole moieties in histidine affinity systems have been widely used for the detection of histidine-containing peptides and biomolecules. The specific interaction between $\mathrm{Ni}^{2+}$ and L-histidine was accomplished through coordination of $\mathrm{Ni}^{2+}$ and the imidazole residue of L-histidine. ${ }^{18-21}$ Here, we used the interaction between $\mathrm{Zn}^{2+}$ and adenosine to develop a novel fluorescence probe for adenosine detection based on beads-QDs-DA. We prepared water-soluble mercaptopropionic acid (MPA)- and mercaptoundecanoic acid (MUA)-capped InP/ZnS QDs. DA was linked to the QD surfaces using a 1-ethyl-3-(3dimethylaminopropyl)carbodiimide/ $N$-hydroxysuccinimide (EDC/NHS) coupling reaction. The PL intensity of QDs-DA is quenched by $\mathrm{Zn}^{2+}$ via strong coordination interactions. In the presence of adenosine, the $\mathrm{Zn}^{2+}$ cations preferentially bind to adenosine, and the QDs-DA PL intensity is recovered.
We constructed a microfluidic chip using a flexible PDMS polymer. The detection of adenosine in the presence of $\mathrm{Zn}^{2+}$ cations was confirmed by fluorescence quenching and recovery of beads-QDs-DA in the microfluidic chip.

\section{Materials and methods Chemicals}

$\mathrm{InP} / \mathrm{ZnS}$ QDs in toluene $(5 \mathrm{mg} / \mathrm{mL}$, emission wavelength $610 \mathrm{~nm}$ ) were purchased from Mesolight (People's Republic of China). Polystyrene/divinyl benzene beads (diameter $30 \mu \mathrm{m}$ ) were purchased from GE Healthcare (Seoul, South Korea). SU-8 2050 and SU-8 developers were purchased from Micro Chem (Newton, MA, USA). PDMS (Sylgard 184) and curing agent were purchased from Dow Corning (Midland, MI, USA). MPA, MUA, L-dopamine hydrochloride, adenosine, EDC, $\mathrm{NHS}, \mathrm{ZnSO}_{4}$, and $n$-hexane were purchased from Sigma-Aldrich (St Louis, MO, USA). Ethanol was purchased from Duksan Pure Chemicals.

Fluorescence emission spectra of the QDs were obtained using a fluoroluminescence spectrometer (Quanta Master, Photon Technology International, NJ, USA) equipped with a xenon lamp (Arc Lamp Housing, A-1010B ${ }^{\mathrm{TM}}$ ), monochromator, and power supply (Brytexbox). Fourier-transform infrared (FTIR) spectra were recorded using a Bruker Vortex 70 FTIR spectrometer. Sonication was performed using a Branson 8510 sonicator. Fluorescence images were obtained using a fluorescence microscope (Nikon, Melville, $\mathrm{NY}$, USA). $\mathrm{O}_{2}$ plasma treatment was performed using plasma cleaner (Harrick Scientific Co., Germany).

\section{Surface modification of $\operatorname{lnP} / \mathrm{ZnS}$ QDs}

An InP/ZnS QD solution $(100 \mu \mathrm{L})$ was added to $2 \mathrm{~mL}$ of $n$-hexane and ethanol, and the mixture was centrifuged at $3,000 \mathrm{rpm}$ for 15 minutes. The precipitate was washed with ethanol, and the centrifugation was repeated twice. The precipitate was collected and redissolved in $n$-hexane $(2 \mathrm{~mL})$. An excess of thiol-terminated MPA and MUA was added to the QD solution. The mixture was sonicated for 30 minutes and kept at room temperature for overnight. The mixture was then centrifuged at 5,000 rpm for 5 minutes and washed with ethanol. The supernatant was removed, and the residue was dried under vacuum for 1 hour to yield water-soluble MPAand MUA-capped InP/ZnS QDs. The surface-modified QDs $(100 \mu \mathrm{L})$ were treated with EDC $(3.8 \mathrm{mg} / \mathrm{mL}, 50 \mu \mathrm{L})$, NHS $(4.6 \mathrm{mg} / \mathrm{mL}, 50 \mu \mathrm{L})$, and DA $(3.5 \mathrm{mg} / \mathrm{mL}, 50 \mu \mathrm{L})$ in phosphate buffer for 3 hours continuous stirring at room temperature to produce QDs-DA. The obtained QDs-DA was purified by precipitation with addition of excess phosphate buffer to the reaction system, and the purified QDs-DA powder was 
redissolved in aqueous solution and stored in a darkroom. These coupling reagents caused reaction of the activated carboxylic acid groups of MPA and MUA with the amine functional group of DA, to produce amide linkages between DA and MPA or MUA on the QDs. The DA-functionalized $\mathrm{InP} / \mathrm{ZnS}$ QDs were conjugated with polystyrene microbeads with amine functional groups through an EDC/sulfo-NHS coupling reaction using a previously described method. ${ }^{22}$

\section{Fabrication of PDMS microfluidic chip with beads-QDs-DA}

A PDMS microfluidic chip with a line of pillars was fabricated using a protocol described in a previous paper. ${ }^{23} \mathrm{SU}-8$ masters were prepared via a photoresist process, and the microfluidic chip was prepared by thermal curing of PDMS. A PDMS slide with channels and holes was attached to a glass slide by $\mathrm{O}_{2}$ plasma treatment. The surfaces of the PDMS microfluidic chip and glass slides were both activated by $\mathrm{O}_{2}$ plasma, using plasma cleaner, at $10.5 \mathrm{~W}$ for 1 minute. After the plasma treatment, they were bonded in an oven at $80^{\circ} \mathrm{C}$ for 40 minutes.
The punched holes on the channel were used as the inlet and outlet, and the silicon tubes (OD $1.6 \mathrm{~mm}$ and OD $0.8 \mathrm{~mm}$ ) were inserted to introduce beads-QDs-DA into the channel. The beads with QDs were packed into a microchannel blocked by pillars using syringe pump. Since the size of the beads is larger than the space between pillars, the beads could not penetrate the pillars in the microchannel of the PDMS chip. The flow rate of the solution was kept at $50 \mu \mathrm{L} / \mathrm{min}$ using a syringe pump. The fluorescence intensity was measured by image scanning over specific areas of the channel. After injection of $\mathrm{Zn}^{2+}$ solution, the fluorescence images on the microchannel were quantified by using image analysis program (IM-1 2005) Ratio Fluorescence Imaging System (PTI), equipped with a xenon lamp and a fluorescent microscope (Olympus IX71).

\section{Results and discussion}

In this study, MPA- and MUA-capped InP/ZnS QDs were prepared, and DA was linked to the QD surfaces and PL quenching as shown in Figure 1. In Figure 1A, paths $i$ and ii show schematic representation of PL quenching and recovery

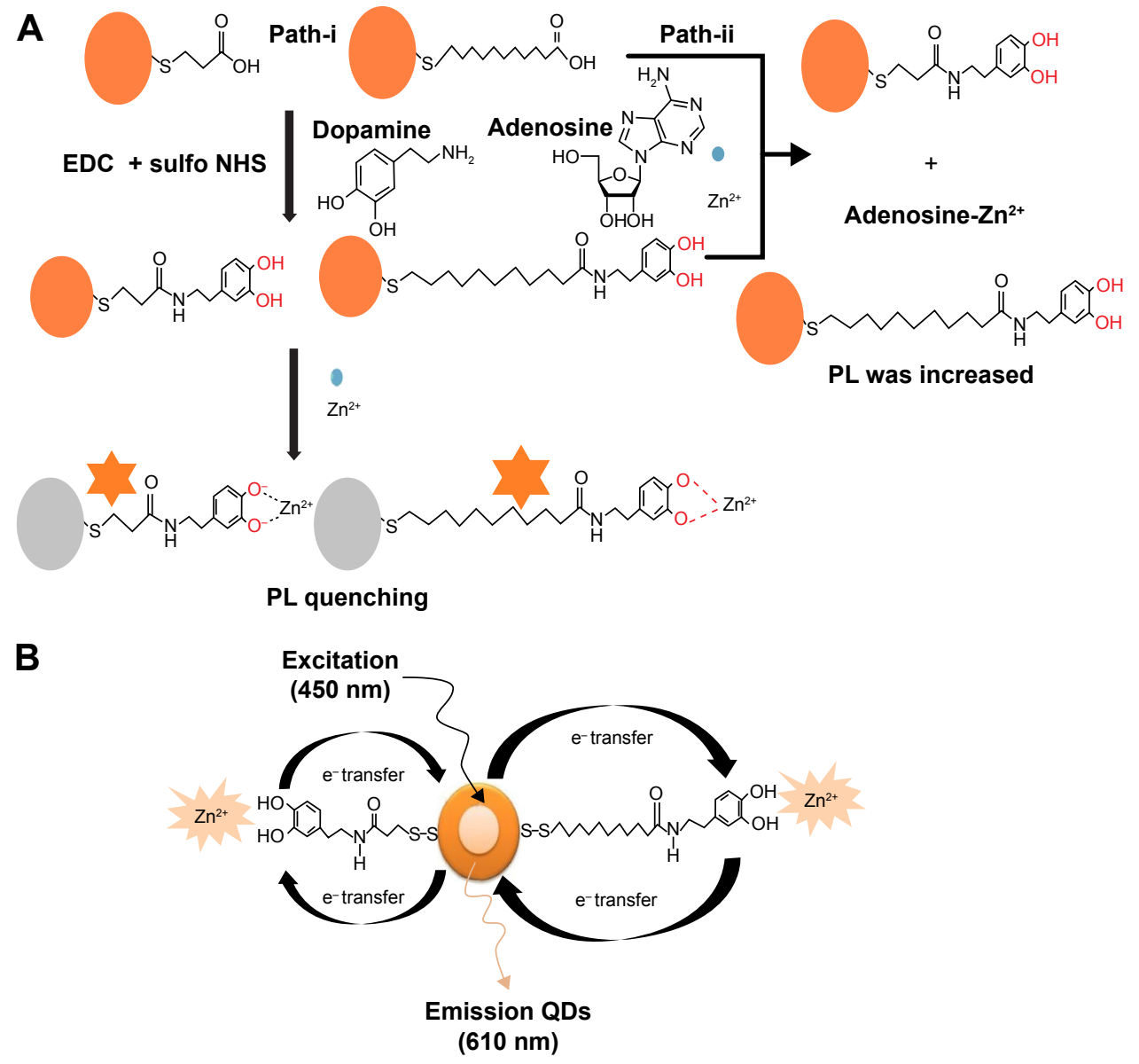

Figure I Schematic diagrams of $(\mathbf{A})$ preparation of water-soluble MPA- and MUA-capped InP/ZnS QDs and (B) fluorescence quenching and recovery by electron transfer. Abbreviations: MPA, mercaptopropionic acid; MUA, mercaptoundecanoic acid; QDs, quantum dots; EDC, I-ethyl-3-(3-dimethylaminopropyl)carbodiimide; NHS, $\mathrm{N}$-hydroxysuccinimide; PL, photoluminescence. 
by the addition of $\mathrm{Zn}^{2+}$ cations and adenosine, respectively, to the system. The PL intensity of the DA-QDs is quenched by $\mathrm{Zn}^{2+}$ because of the strong coordination interactions between $\mathrm{DA}$ and $\mathrm{Zn}^{2+} . \mathrm{Zn}^{2+}$ ions induce electron transfer from the QDs, and PL quenching occurs. In the presence of adenosine, the $\mathrm{Zn}^{2+}$ cations preferentially bind to adenosine, and electron transfer from the QDs decreases; therefore, the PL intensity of the QDs-DA is recovered. The PL quenching and recovery also depend on the chain length of the organic ligand on the QD surfaces. MPA and MUA both were thiol-substituted carboxylic acids with three and eleven carbon chain lengths, respectively. Organic QDs surface was modified with this ligand to produce water soluble QDs for biological assays.

Figure 1B shows the representation of PL quenching mechanism. Fluorescence quenching was broadly explained by two possible mechanisms. ${ }^{24}$ One is dynamic quenching, which results from the collision between the fluorophore and a quencher, and the second one is static quenching, which results from the ground-state complex formation between the fluorophore and a quencher. According to studies on the mechanisms of fluorescence quenching of QDs and metal ions, ${ }^{25-27}$ the above process can be attributed to effective electron transfer from the QDs to the $\mathrm{Zn}^{2+}$ ions, and fluorescence recovery can be attributed to effective electron transfer from $\mathrm{Zn}^{2+}$ ions to QDs after addition of adenosine, based on the strong affinity of zinc for nitrogen atoms, which correspondingly yields the nonradiative recombination of excited electrons $\left(\mathrm{e}^{-}\right)$in the conduction bands and holes $\left(\mathrm{h}^{+}\right)$in the valence band on the surface of QDs.

Figure $2 \mathrm{~A}$ and $\mathrm{B}$ shows photographs of the photomask for the photoresist process for SU-8 molding and the fabricated PDMS microfluidic chip on the glass slide by using $\mathrm{O}_{2}$ plasma, respectively. The chip $(25 \mathrm{~mm} \times 12 \mathrm{~mm})$ was

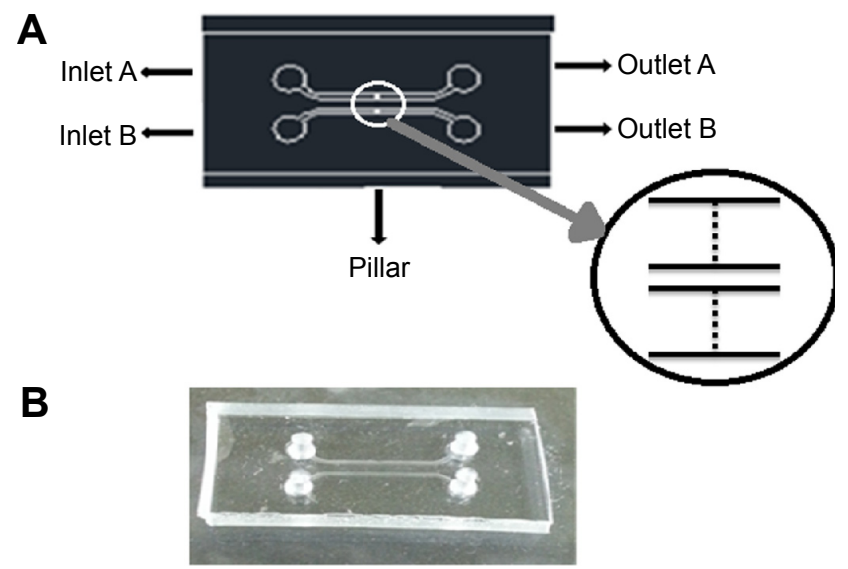

Figure 2 Photographs of (A) photomask for photoresist process for SU-8 molding, inset photo shows the amplified image of pillars in the channel, and (B) fabricated PDMS microfluidic chip on glass slide by using $\mathrm{O}_{2}$ plasma.

Abbreviation: PDMS, polydimethylsiloxane. fabricated using a conventional photo process. The channel width and height were $400 \mu \mathrm{m}$ and $50 \mu \mathrm{m}$, respectively, and the distance between pillars was $25 \mu \mathrm{m}$. The elliptical pillar was constructed with a long diameter of $100 \mu \mathrm{m}$ and a short diameter of $50 \mu \mathrm{m}$. The chip had two inlets and two outlets, with two channels. The channels were packed with the beadsQDs-DA. Figure 2A inset photo shows the amplified image of pillars in the channel. The gaps between the pillars were small enough to block the beads but large enough for buffer solution to flow through the channels.

Figure $3 \mathrm{~A}-\mathrm{C}$ shows the FTIR spectra of MPA, L-DA, and beads-QDs-DA, respectively. The peak at $2,580 \mathrm{~cm}^{-1}$ in (A) arises from the $\mathrm{S}-\mathrm{H}$ stretching vibration of MPA. This peak is not observed in (C), which confirms covalent bond formation between the thiol group of MPA and the metal atom in the QD. The peaks at $1,654 \mathrm{~cm}^{-1}$ and $2,978 \mathrm{~cm}^{-1}$ in (A) are attributed to the $\mathrm{C}=\mathrm{O}$ stretching of carboxylic groups and $\mathrm{C}-\mathrm{H}$ stretching of methylene groups, respectively. The peaks at 3,206 and 2,977 $\mathrm{cm}^{-1}$ in (B) are attributed to $\mathrm{N}-\mathrm{H}$ stretching of amine functional groups and $\mathrm{C}-\mathrm{H}$ stretching of aromatic benzene rings, respectively. The peaks at $1,684 \mathrm{~cm}^{-1}$ and $1,610 \mathrm{~cm}^{-1}$ in (C) are attributed to the $-\mathrm{CONH}$ amide band I group and the $\mathrm{N}-\mathrm{H}$ amide band II group, respectively, and the peak at $1,230 \mathrm{~cm}^{-1}$ is attributed to the $-\mathrm{C}-\mathrm{N}$ stretching of amide bond, these arise from conjugation of DA with the MPA-capped QDs.

Figure 4A and B, respectively, shows photographs of the QDs before and after surface modification with MPA under ultraviolet (UV) illumination. The InP/ZnS QDs are dispersed in a two-layered mixture of $n$-hexane (upper) and deionized water (lower). The figure shows that before the surface treatment, the QDs were dispersed in the organic

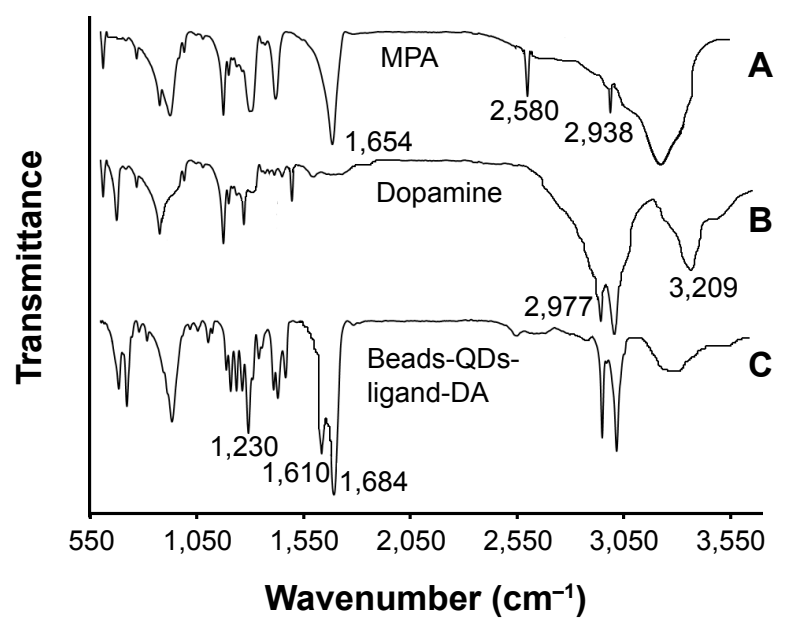

Figure 3 FTIR spectra of (A) MPA, (B) L-DA, and (C) beads-QDs-DA. Abbreviations: FTIR, fourier-transform infrared; MPA, mercaptopropionic acid; DA, dopamine; QDs, quantum dots. 

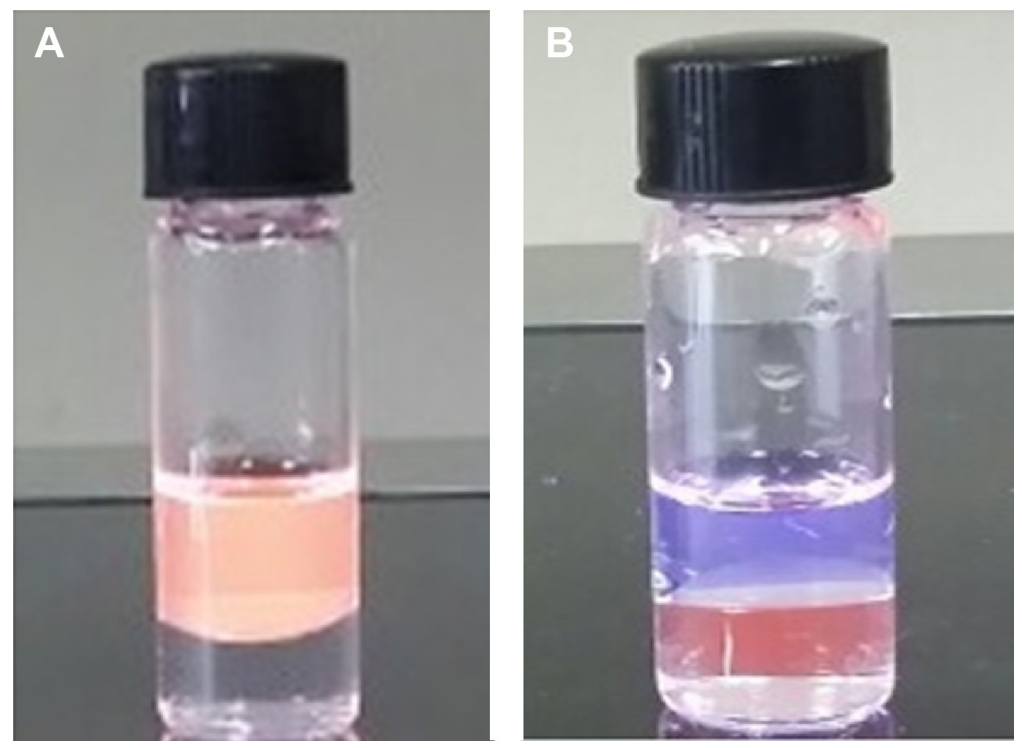

Figure 4 Photographs of QDs (A) before and (B) after surface modification with MPA under UV illumination. Abbreviations: QDs, quantum dots; MPA, mercaptopropionic acid; UV, ultraviolet.

phase, but after treatment with MPA, they were dispersed in the water phase since the MPA ligand was water soluble. These surface modified QDs were used for the detection of adenosine in presence of $\mathrm{Zn}^{2+}$.
Figure 5 shows the fluorescence spectra of the QDs-DA, QDs-DA- $\mathrm{Zn}^{2+}$, and QDs-DA-Zn ${ }^{2+}$-adenosine after surface modification with (A) MPA and (B) MUA. Inset photographs depict the (i) QDs-DA, (ii) QDs-DA- $\mathrm{Zn}^{2+}$, and

A
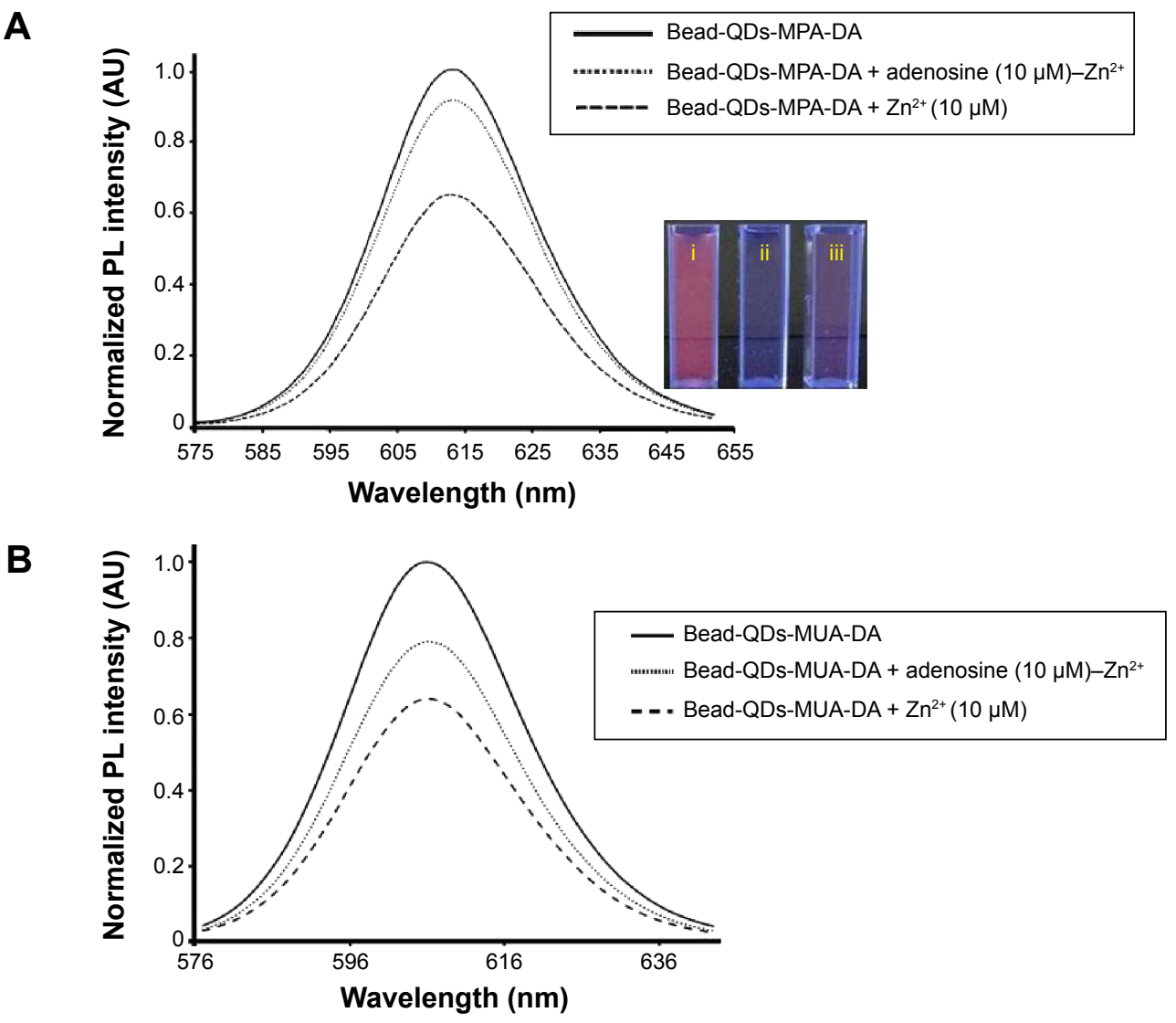

Figure 5 Normalized PL spectra of QDs-DA, QDs-DA-Zn ${ }^{2+}$, and QDs-DA-Zn ${ }^{2+}$-adenosine with surface modification by (A) MPA and (B) MUA. Inset photograph depicts the (i) QDs-DA, (ii) QDs-DA-Zn², and (iii) QDs-DA-Znn ${ }^{2+}$ with adenosine system.

Abbreviations: PL, photoluminescence; QDs, quantum dots; DA, dopamine; MPA, mercaptopropionic acid; MUA, mercaptoundecanoic acid; AU, atomic units. 
(iii) QDs-DA- $\mathrm{Zn}^{2+}$ with adenosine system. After the addition of $10 \mu \mathrm{mol} \mathrm{L}{ }^{-1} \mathrm{Zn}^{2+}$, the PL intensity of the QDs-DA decreased. When $10 \mu \mathrm{mol} \mathrm{L^{-1 }}$ adenosine was added to the QDs-DA-Zn ${ }^{2+}$ system, the PL intensity increased significantly. The PL intensity was quenched by $\sim 62 \%$ by electron transfer from the QDs to the $\mathrm{Zn}^{2+}$ cation; $90 \%$ of the PL intensity was recovered in the case of beads-QDs-MPA-DA and 78\% in the case of beads-QDs-MUA-DA after addition of adenosine, as a result of preferential binding of $\mathrm{Zn}^{2+}$ to adenosine.
Figure 6 shows the fluorescence microscope images of beads-QDs-DA in the microfluidic channel under (A) bright field and (B) dark field conditions, and (C) as a function of time after introduction of $\mathrm{Zn}^{2+}$ solution. The figure shows that the QDs are well bound to the polystyrene bead surfaces, and the microfluidic chip channels are filled with beads-QDs-DA. All reaction solutions were injected into the microchannel by using syringe pump. Initially, the microchannel was washed with DI water, and $\mathrm{Zn}^{2+}$ and adenosine

A

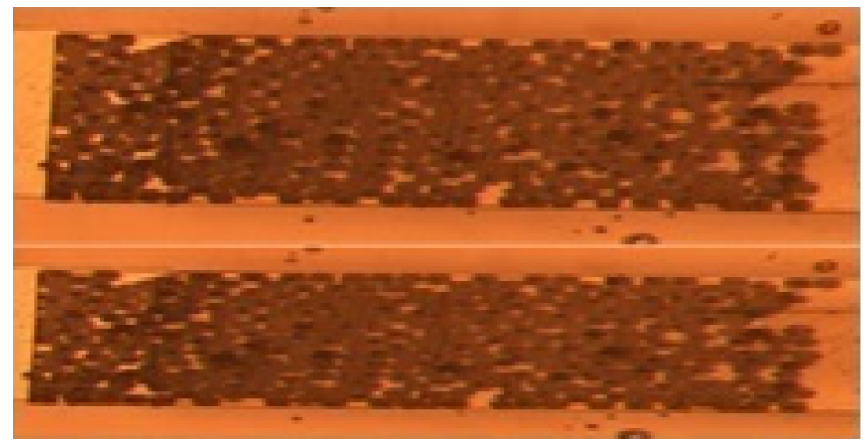

B

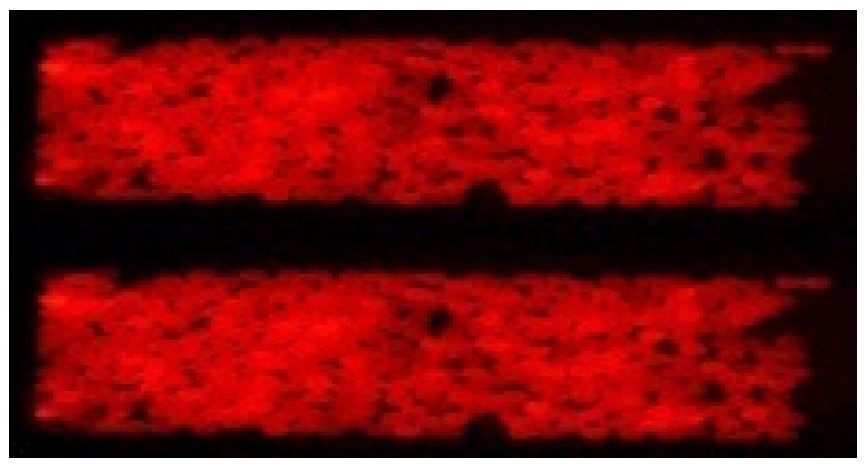

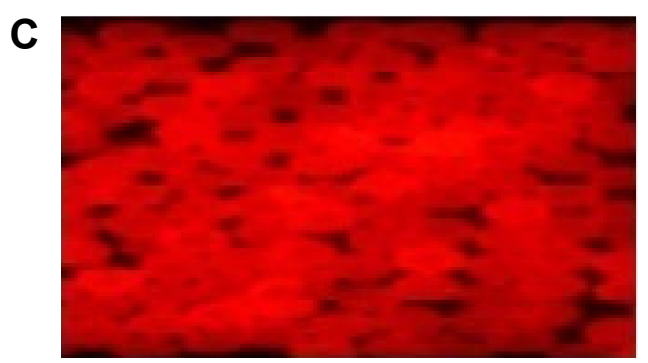

$0 \mathrm{~min}$

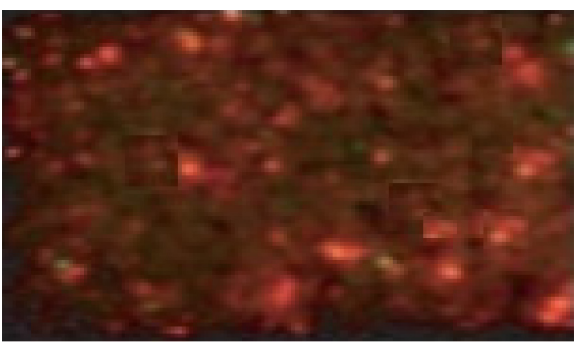

$20 \mathrm{~min}$

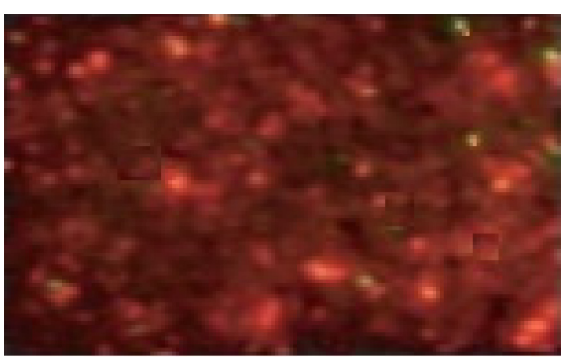

$10 \min$

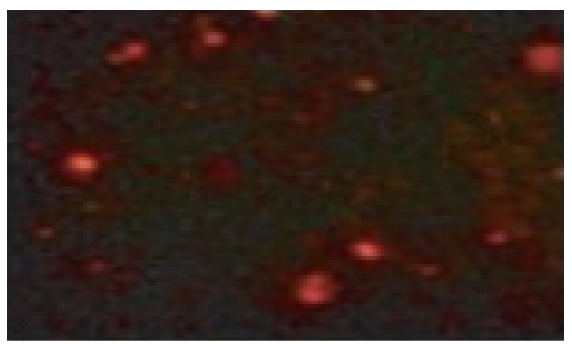

$30 \mathrm{~min}$

Figure 6 Fluorescence microscope images of beads-QDs-DA in microfluidic channel under (A) bright field and (B) dark field conditions, and (C) beads-QDs-DA packed in PDMS chip as a function of time after introduction of $\mathrm{Zn}^{2+}$ solution.

Abbreviations: DA, dopamine; QDs, quantum dots; PDMS, polydimethylsiloxane; min, minutes. 


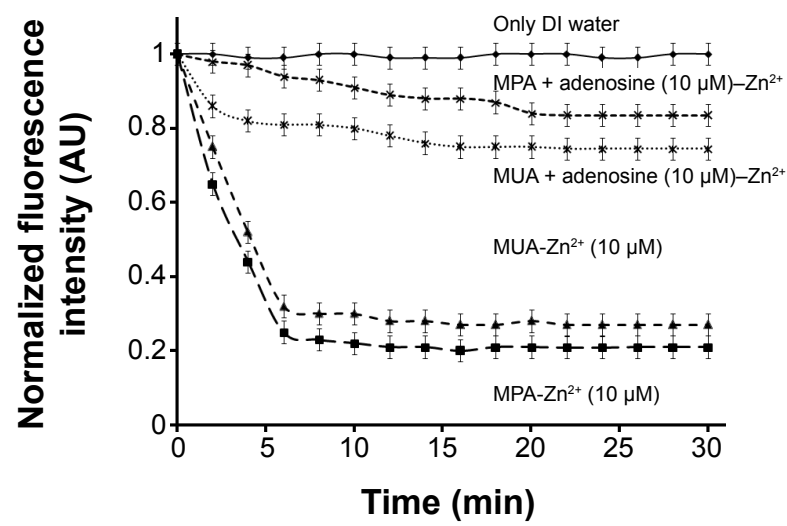

Figure 7 Fluorescence intensity variations of QDs as a function of time after injection of $10 \mu \mathrm{mol} \mathrm{L} \mathrm{L}^{-1} \mathrm{Zn}^{2+}$ solution.

Abbreviations: QDs, quantum dots; MPA, mercaptopropionic acid; MUA, mercaptoundecanoic acid; min, minutes; DI, deionized.

were injected into the microchannel for 30 minutes. After injection of $\mathrm{Zn}^{2+}$ using a syringe pump, the fluorescence microscope images were recorded as a function of time. The fluorescence intensity was measured by image scanning over specific areas of the channel. After injection of $\mathrm{Zn}^{2+}$ solution, the fluorescence images on the microchannel were quantified by using image analysis program (IM-1 2005) and redrawn as intensity line profile and also the fluorescence intensity per minute at 360 pixel of line profiles were averaged and converted to intensity profile as a function of time as shown in Figure 6. The fluorescence emission intensity from QDs attached to microbeads decreased gradually due to strong affinity between $\mathrm{Zn}^{2+}$ and adenosine. After 30 minutes, it was very difficult to observe further change of the fluorescence intensity, which may be due to the saturation of preferential binding of $\mathrm{Zn}^{2+}$ to adenosine.

Figure 7 shows the fluorescence intensity variations of the QDs as a function of time after $1.5 \mathrm{~mL}$ injection of $10 \mu \mathrm{mol} \mathrm{L} \mathrm{L}^{-1} \mathrm{Zn}^{2+}$ and adenosine solution. The flow rate was kept at $50 \mu \mathrm{L} / \mathrm{min}$ using a syringe pump. After addition of $10 \mu \mathrm{mol} \mathrm{L}^{-1} \mathrm{Zn}^{2+}$, the PL intensity of the QDs-DA gradually decreased within 30 minutes and the fluorescence was significantly recovered by the addition of $10 \mu \mathrm{mol} \mathrm{L}^{-1}$ adenosine. This experiment results also correlated with fluorescence spectra. The PL intensity was quenched by $\sim 60 \%-65 \%$ by electron transfer from the QDs to the $\mathrm{Zn}^{2+}$ cation; $85 \%$ of the PL intensity was recovered in the case of beads-QDsMPA-DA and 75\% in the case of beads-QDs-MUA-DA after addition of adenosine, as a result of preferential binding of $\mathrm{Zn}^{2+}$ to adenosine. The figure also shows that for a shorter chain length between $\mathrm{Zn}^{2+}$ and the QDs (MPA), PL quenching was faster. The recovery of PL intensity was higher for a shorter chain length and vice versa. The experiment was repeated three times, with the fluorescence intensity vs time expressed as the mean \pm standard deviation, as shown in Figure 7.

\section{Conclusion}

In summary, water-soluble InP/ZnS QDs were prepared by surface modification with MPA and MUA ligands. The QDs were immobilized on polystyrene microbeads by an EDC/sulfo-NHS coupling reaction, and DA was conjugated with the QDs. Fluorescence quenching and recovery were observed as a result of electron-transfer interactions between DA and $\mathrm{Zn}^{2+}$. A PDMS microfluidic chip was fabricated with polystyrene beads-QDs-DA packed in the channel. After addition of $\mathrm{Zn}^{2+}$, the PL intensity of the QDs-DA decreased and was recovered by addition of adenosine. A shorter chain length between $\mathrm{Zn}^{2+}$ and the QDs gave faster PL intensity quenching and recovery. The proposed fluorescence bioprobe has potential application in biological systems for the detection of adenosine in solution and serum at low concentration levels.

\section{Acknowledgment}

This work was supported by a National Research Foundation (NRF) grant funded by the Korean government (MEST) (NRF-2006-2005382).

\section{Disclosure}

The authors report no conflicts of interest in this work.

\section{References}

1. Craig CG, Temple SD, White TD. Is cyclic AMP involved in excitatory amino acid-evoked adenosine release from rat cortical slices? Eur J Pharmacol. 1994;269:79-85.

2. Fredholm BB, IJzerman AP, Jacobson KA, Linden J, Müller CE. International union of basic and clinical pharmacology. LXXXI. Nomenclature and classification of adenosine receptors - an update. Pharmacol Rev. 2011; 63:1-34.

3. Pijanowska DG, Remiszewska E. pH-based detection of phenylalnine by potentiometric and colorimetric methods. Sensors. 2006;6:428-434.

4. Hu YF, Zhang ZH, Zhang HB, Luo LJ, Yao SZ. Electrochemical determination of 1-phenylalanine at polyaniline modified carbon electrode based on $\beta$-cyclodextrin incorporated carbon nanotube composite material and imprinted sol-gel film. Talanta. 2011;84:305-313.

5. Kand'ár R, Žáková P. Determination of phenylalanine and tyrosine in plasma and dried blood samples using HPLC with fluorescence detection. J Chromatogr B. 2009;877:3926-3929.

6. Wróbel K, Wróbel K. Determination of aspartame and phenylalanine in diet soft drinks by high-performance liquid chromatography with direct spectrofluorimetric detection. J Chromatogr A. 1997;773:163-168.

7. Tagliaro F, Moretto S, Valentini R, et al. Capillary zone electrophoresis determination of phenylalanine in serum: a rapid, inexpensive and simple method for the diagnosis of phenylketonuria. Electrophoresis. 1994; 15:94-97.

8. Abbasi F, Mirzadeh H, Katbab A-A. Modification of polysiloxane polymers for biomedical applications: a review. Polym Int. 2001;50: 1279-1287.

9. Owen MJ, Smith PJ. Plasma treatment of polydimethylsiloxane. J Adhes Sci Technol. 1994;8:1063-1075. 
10. Lucas LJ, Chesler JN, Yoon J-Y. Lab-on-a-chip immunoassay for multiple antibodies using microsphere light scattering and quantum dot emission. Biosens Bioelectron. 2007;23:675-681.

11. Sato K, Tokeshi M, Odake T, et al. Integration of an immunosorbent assay system: analysis of secretory human immunoglobulin A on polystyrene beads in a microchip. Anal Chem. 2000;72:1144-1147.

12. Baron R, Zayats M, Willner I. Dopamine-, 1-DOPA-, adrenaline-, and noradrenaline-induced growth of Au nanoparticles: assays for the detection of neurotransmitters and of tyrosinase activity. Anal Chem. 2005;77: $1566-1571$

13. Brown AS, Gershon S. Dopamine and depression. JNeural Transm. 1993; 91:75-109.

14. Bharali DJ, Lucey DW, Jayakumar H, Pudavar HE, Prasad PN. Folatereceptor-mediated delivery of InP quantum dots for bioimaging using confocal and two-photon microscopy. J Am Chem Soc. 2005;127: 11364-11371.

15. Kang I, Wise FW. Electronic structure and optical properties of $\mathrm{PbS}$ and PbSe quantum dots. J Opt Soc Am B. 1997;14:1632-1646.

16. Rogach A, Kershaw S, Burt M, et al. Colloidally prepared HgTe nanocrystals with strong room-temperature infrared luminescence. Adv Mater. 1999;11:552-555.

17. Du H, Chen CL, Krishnan R, et al. Optical properties of colloidal PbSe nanocrystals. Nano Lett. 2002;2:1321-1324.

18. Fraser KA, Harding MM. The crystal and molecular structure of bis(histidino)nickel(II) monohydrate. J Chem Soc A. 1967:415-420.

19. Liu Y, Li Y, Wu ZQ, Yan XP. Fabrication and characterization of hexahistidine-tagged protein functionalized multi-walled carbon nanotubes for selective solid-phase extraction of $\mathrm{Cu}^{2+}$ and $\mathrm{Ni}^{2+}$. Talanta. 2009; 79:1464-1471.
20. Liu JW, Yang T, Ma LY, Chen XW, Wang JH. Nickel nanoparticle decorated graphene for highly selective isolation of polyhistidine-tagged proteins. Nanotechnology. 2013;24:505704-505711.

21. Liu J-W, Yang T, Chen S, Chen XW, Wang JH. Nickel chelating functionalization of graphene composite for metal affinity membrane isolation of lysozyme. J Mater Chem B. 2013;1:810-818.

22. Yoo JH, Jongsung K. The specific hybridization of $\mathrm{p} 53$ gene on beadquantum dot complex in microfluidic chip. JNanosci Nanotechnol. 2011; 11:7082-7085.

23. Yoo JH, Jongsung K. Identification of p53 gene by using $\mathrm{CdSe} / \mathrm{ZnS}$ conjugation and hybridization. J Nanosci Nanotechnol. 2011;11: 4343-4346.

24. Lakowicz JR, Weber G. Quenching of fluorescence by oxygen. Probe for structural fluctuations in macromolecules. Biochemistry. 1973; 12:4161-4170.

25. Wang J, Liang J, Sheng Z. A novel strategy for selective detection of $\mathrm{Ag}+$ based on the red-shift of emission wavelength of quantum dots. Microchim Acta. 2009;167:281-287.

26. Cai ZX, Yang H, Zhang Y. Preparation, characterization and evaluation of water-soluble 1-cysteine-capped-CdS nanoparticles as fluorescence probe for detection of $\mathrm{Hg}(\mathrm{II})$ in aqueous solution. Anal Chim Acta. 2006;559:234-239.

27. Chen J, Gao YC. A, novel fluorescent array for mercury(II) ion in aqueous solution with functionalized cadmium selenide nanoclusters. Anal Chim Acta. 2006;577:77-84.
International Journal of Nanomedicine

\section{Publish your work in this journal}

The International Journal of Nanomedicine is an international, peerreviewed journal focusing on the application of nanotechnology in diagnostics, therapeutics, and drug delivery systems throughout the biomedical field. This journal is indexed on PubMed Central, MedLine, CAS, SciSearch ${ }^{\circledR}$, Current Contents ${ }^{\circledR} /$ Clinical Medicine,

\section{Dovepress}

Journal Citation Reports/Science Edition, EMBase, Scopus and the Elsevier Bibliographic databases. The manuscript management system is completely online and includes a very quick and fair peer-review system, which is all easy to use. Visit http://www.dovepress.com/ testimonials.php to read real quotes from published authors. 